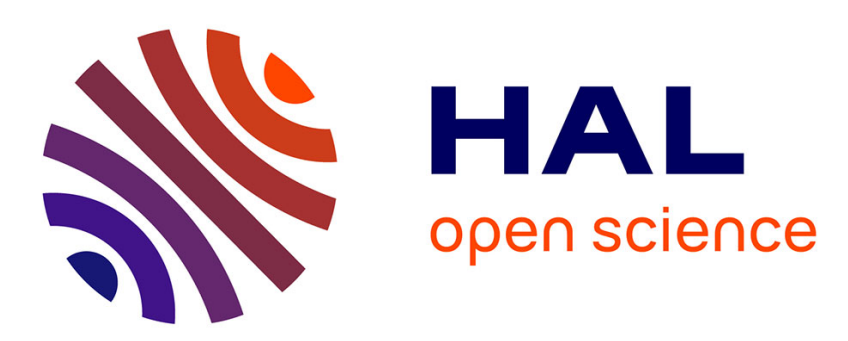

\title{
Size dependence of the polarizability and Haynes rule for an exciton bound to an ionized donor in a single spherical quantum dot
}

\author{
E. Feddi, A. Zouitine, A. Oukerroum, F. Dujardin, E. Assaid, M. Zazoui
}

\section{- To cite this version:}

E. Feddi, A. Zouitine, A. Oukerroum, F. Dujardin, E. Assaid, et al.. Size dependence of the polarizability and Haynes rule for an exciton bound to an ionized donor in a single spherical quantum dot. Journal of Applied Physics, 2015, 117 (6), pp.064309. 10.1063/1.4907760 . hal-01517442

\section{HAL Id: hal-01517442 \\ https://hal.univ-lorraine.fr/hal-01517442}

Submitted on 11 May 2017

HAL is a multi-disciplinary open access archive for the deposit and dissemination of scientific research documents, whether they are published or not. The documents may come from teaching and research institutions in France or abroad, or from public or private research centers.
L'archive ouverte pluridisciplinaire HAL, est destinée au dépôt et à la diffusion de documents scientifiques de niveau recherche, publiés ou non, émanant des établissements d'enseignement et de recherche français ou étrangers, des laboratoires publics ou privés. 
Size dependence of the polarizability and Haynes rule for an exciton bound to an ionized donor in a single spherical quantum dot

E. Feddi' , A. Zouitine, A. Oukerroum, F. Dujardin, E. Assaid, and M. Zazoui

Citation: Journal of Applied Physics 117, 064309 (2015); doi: 10.1063/1.4907760

View online: http://dx.doi.org/10.1063/1.4907760

View Table of Contents: http://aip.scitation.org/toc/jap/117/6

Published by the American Institute of Physics

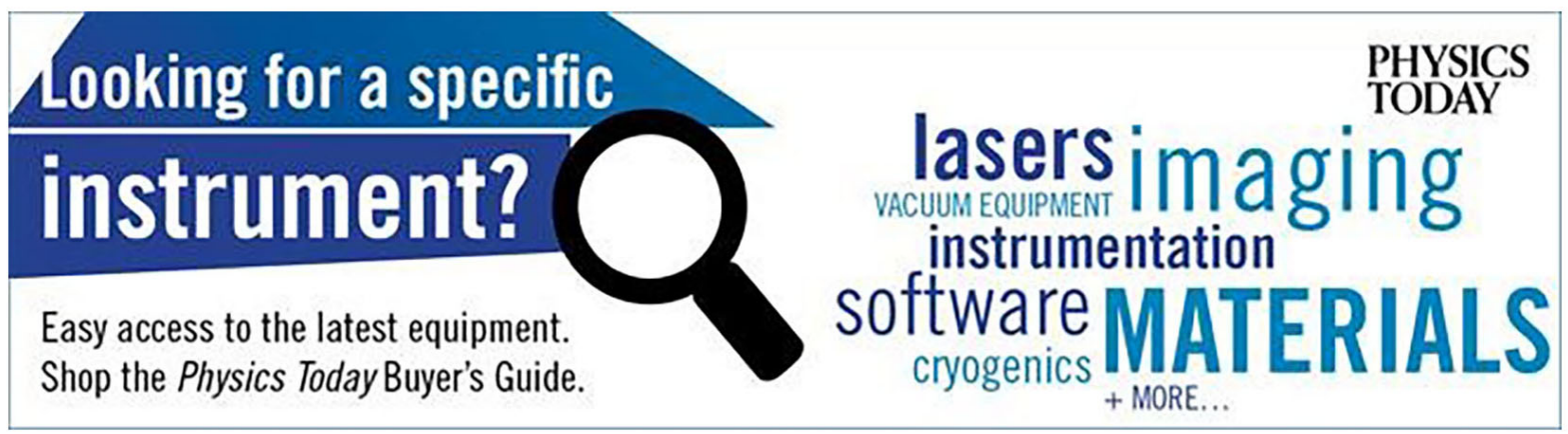




\title{
Size dependence of the polarizability and Haynes rule for an exciton bound to an ionized donor in a single spherical quantum dot
}

\author{
E. Feddi, ${ }^{1, a)}$ A. Zouitine,${ }^{1}$ A. Oukerroum, ${ }^{2}$ F. Dujardin, ${ }^{3}$ E. Assaid, ${ }^{4}$ and M. Zazoui ${ }^{2}$ \\ ${ }^{1}$ Université Mohamed V Souissi, Ecole Normale Supérieure de l'Enseignement Technique (ENSET), Rabat, \\ Morocco \\ ${ }^{2}$ Laboratory of Condensed Matter, Faculty of Sciences and Techniques, University of Hassan II-Mohammedia, \\ B.P. 146, 20800 Mohammedia, Morocco \\ ${ }^{3}$ LCP-A2MC, Université de Lorraine, ICPM, 1 Bd Arago, 57070 Metz, France \\ ${ }^{4}$ Laboratoire d'Électronique et Optique des Nanostructures de Semiconducteurs, Faculté des Sciences, \\ B. P. 20, El Jadida, Morocco
}

(Received 2 November 2014; accepted 28 January 2015; published online 10 February 2015)

\begin{abstract}
We study the effect of an external electric field on an exciton bound to an ionized donor $\left(D^{+}, X\right)$ confined in a spherical quantum dot using a perturbative-variational method where the wave function and energy are developed in series of powers of the electric field strength. After testing this new approach in the determination of the band gap for some semiconductor materials, we generalize it to the case of $\left(D^{+}, X\right)$ in the presence of the electric field and for several materials $\mathrm{ZnO}, \mathrm{PbSe}$, and $I n A s$, with significant values of the mass ratio. Three interesting results can be deduced: First, we show that the present method allows to determine the ground state energy in the presence of a weak electric field in a simple way $\left(E=E_{0}-\alpha f^{2}\right)$ using the energy without electric field $E_{0}$ and the polarizability $\alpha$. The second point is that our theoretical predictions show that the polarizability of $\left(D^{+}, X\right)$ varies proportionally to $R^{3.5}$ and follows an ordering $\alpha_{D^{0}}<\alpha_{X}<\alpha_{\left(D^{+}, X\right)}$. The last point to highlight is that the Haynes rule remains valid even in the presence of a weak electric field. (C) 2015 AIP Publishing LLC. [http://dx.doi.org/10.1063/1.4907760]
\end{abstract}

\section{INTRODUCTION}

The great advance in semiconductor growth techniques has permitted to fabricate quantum structures with various shapes, where the electrons $(e)$, holes $(h)$, and excitons $(X)$ are confined in $2 D, 1 D$, or completely in $0 D$. The confinement effect leads to the quantization of electronic states, an enhancement of electron-hole orbitals overlap, and a strengthening of the exciton binding energy. During the growth process, it is possible intentionally or unintentionally to add impurities into the nano dots. This inclusion causes an additional change in the optical properties of quantum structures because excitons can easily be bounded to the donor $\left(D^{0}, X\right)$ (acceptor $\left.\left(A^{0}, X\right)\right)$ or to an ionized donor $\left(D^{+}, X\right)$ (acceptor $\left(A^{-}, X\right)$ ). The binding energies of all of these complexes are enhanced in confined systems that are much more stable than in the bulk semiconductor materials which facilitate their experimental observations.

Since its prediction in 3D semiconductors, ${ }^{1}$ it is well known that the exciton bound to an ionized donor $\left(D^{+}, X\right)$ is stable only for materials having an electron-to-hole effective mass ratio $\sigma=m_{e}^{*} / m_{h}^{*}$ less than a critical value $\sigma_{c}=0.426{ }^{2}$ In two-dimensional semiconductors, this complex remains stable for $\sigma \leq \sigma_{c}=0.88$, which is a value about two times larger than that obtained in 3D case due to the confinement effect. ${ }^{3,4}$ In low dimensional semiconductors, the $\left(D^{+}, X\right)$ complex has been the subject to considerable investigations: in a spherical quantum dot (SQD), Stébé et al. ${ }^{5}$ have

\footnotetext{
${ }^{\text {a) }}$ Author to whom correspondence should be addressed. Electronic mail: e.feddi@um5s.net.ma
}

theoretically studied the electronic and optical properties of $\left(D^{+}, X\right)$ as a function of the sphere radius. They have shown that the quantum confinement gives rise to "giant" oscillator strength compared with the bulk semiconductor case so that the optical transitions involving excitons bound to impurities can be observed more easily in confined systems. Fonoberov and Balandin ${ }^{6-8}$ have investigated the origin of ultraviolet photo-luminescence (UV-PL) in $\mathrm{ZnO}$ quantum dots by using a multi-band effective-mass method and by taking into account the electron and hole self-energies. Their results show that the major source of UV-PL is either recombination of confined excitons or surface-bound ionized acceptor exciton complexes. Baskoutas et al. ${ }^{9}$ have calculated the binding energy and recombination rate for $\left(D^{+}, X\right)$ in a two dimensional parabolic quantum dot by using the Hartree formalism with Potential Morphing Method (PMM). Their results show that $\left(D^{+}, X\right)$ becomes unstable for $R$ lower than a critical radius $R_{c}$, which depends on the impurity position. Xie and $\mathrm{Gu}^{10}$ had demonstrated that the stability is obtained for all values of electron to hole mass ratio. Avetisyan et al. ${ }^{11}$ have treated this problem for the parabolic confinement. Recently, the experimental studies in $\mathrm{ZnO}^{12}$ have allowed to obtain the Haynes rule where the linear dependences on the donor binding energy $E_{D}$ for both neutral $\left(D^{0}, X\right)$ and ionized donor bound exciton $\left(D^{+}, X\right)$ are given by $E_{\left(D^{0} . X\right)}=0.37 E_{D}$ $-4.2(\mathrm{meV})$ and $E_{\left(D^{+}, X\right)}=0.5 E_{D}-23(\mathrm{meV})$.

The electric field effect on the low dimension semiconductors continues to attract much interest. ${ }^{13-21}$ It leads to the quantum confined Stark effect characterized by a red-shift, many times greater than the electron-hole binding energy. 
However, very few studies were devoted to the influence of electric field on the $\left(D^{+}, X\right)$ complex. We cite three interesting theoretical reports concerned with the study of the effect of an applied electric field on $\left(D^{+}, X\right)$ in low dimension semiconductors. Niculescu et $a l .{ }^{22}$ have used a simple model based on the variational-perturbation procedure in order to investigate the ground state of $\left(D^{+}, X\right)$ in a parabolic quantum well using a screened potential for the hole-ionized donor interaction. They have found that the electric field breaks down the degeneracy of states for the impurities symmetrically positioned in the well, and they have shown that the existence of bound states depends on screened potential and electric field strength. In one of our papers, ${ }^{23}$ we have studied the competition between the confinement effect and the Stark effect as function of the spherical dot size and the applied electric field strength. We have shown that for intermediate and low confinement, the exciton is less sensitive to the effect of the applied electric field than the $\left(D^{+}, X\right)$ complex. Indeed, the presence of the ionized donor leads to an easier spatial separation of electron and hole orbitals, which reduces the overlap of the electron and hole wave functions due to the ionized donor $D^{+}$and accelerates the dissociation process of exciton for low quantum confinement regime, when the SQDs are submitted to an electric field. More recently, Shi and $\mathrm{Yan}^{24}$ have shown that within the electric field, the energy of $\left(D^{+}, X\right)$ depends on the electric field and the shape of the ellipsoidal quantum dots. Their results show that the influence of the dot shape is more important in the case of the light hole. On the other hand, the effect the electric field becomes more pronounced for the heavy hole.

The purpose of this paper is to determine the ground state energy of an exciton bound to an ionized donor in an isotropic SQD submitted to an electric field. Calculations will be performed in the framework of effective mass approximation using a variational-perturbative method and by choosing an appropriate wave function taking into account different inter-particle correlations and developed in a series of electric field powers. This approach was successfully used in the past by Atanasoff in order to determine the dielectric constant of Helium ${ }^{25}$ and by Feddi et al. to study the Stark shift of negatively charged exciton ${ }^{26}$ and donor impurity. ${ }^{17}$ In Sec. II, we present the development of our theoretical formalism. The Hamiltonian of the system is developed in Hylleras coordinates and the Schrödinger equation is then solved numerically. In Sec. III, we apply our model to the exciton case and we compare the results obtained with some theoretical and experimental data. Then we generalize our model to the $\left(D^{+}, X\right)$ complex and we suggest some predictions of the behavior of the confined Stark shift and the polarizability.

\section{THEORETICAL BACKGROUND}

Let us consider an exciton $X=(e, h)$ bound to an ionized donor $D^{+}$placed at the center of an isotropic SQD. The system is submitted to a uniform electric field $\vec{F}$ directed along the z-axis. In the envelope wave function approximation, the Hamiltonian reads

$$
H=H_{0}+W,
$$

where $W$ is the electric energy operator and $H_{0}$ denotes the Hamiltonian of the unperturbed system given by

$$
\begin{aligned}
H_{0} & =T_{e}+T_{h}+V_{c}+V_{w} \\
& =-\frac{1}{2} \Delta_{e}-\frac{\sigma}{2} \Delta_{h}+\left(\frac{1}{r_{h}}-\frac{1}{r_{e}}-\frac{1}{r_{e h}}\right)+V_{w} .
\end{aligned}
$$

$T_{e}$ and $T_{h}$ are, respectively, the electron and hole kinetic energies, $V_{c}=\frac{1}{r_{h}}-\frac{1}{r_{e}}-\frac{1}{r_{e h}}$ is the Coulomb potential, and $V_{w}$ the confinement potential. $\sigma=m_{e}^{*} / m_{h}^{*}$ is the electron-to-hole effective masses ratio, $r_{e}$ and $r_{h}$ are the electron and hole coordinates, and $r_{e h}$ the electron-hole distance. We have used effective atomic units with a length equal to the $3 D$ donor effective Bohr radius $a^{*}=\varepsilon \hbar^{2} /\left(e^{2} m_{e}^{*}\right)$ and an energy equal to the Hartree energy $R^{*}=\hbar^{2} /\left(m_{e}^{*} a^{* 2}\right)$.

Because the axial symmetry around the z-axis, we introduce the Hylleraas coordinates $\left(r_{e}, r_{h}, r_{e h}, z_{e}, z_{h}\right) .{ }^{27}$ Thus, the electron kinetic energy operator can be written as

$$
\begin{aligned}
T_{e}= & -\frac{1}{2} \frac{\partial^{2}}{\partial r_{e}^{2}}-\frac{1}{2} \frac{\partial^{2}}{\partial r_{e h}^{2}}-\frac{1}{2}\left(\frac{r_{e}^{2}-r_{h}^{2}+r_{e h}^{2}}{r_{e} r_{e h}}\right) \frac{\partial^{2}}{\partial r_{e} \partial r_{e h}}-\frac{1}{r_{e}} \frac{\partial}{\partial r_{e}} \\
& -\frac{1}{r_{e h}} \frac{\partial}{\partial r_{e h}}-\frac{z_{e}}{r_{e}} \frac{\partial^{2}}{\partial z_{e} \partial r_{e}}-\left(\frac{z_{e}-z_{h}}{r_{e h}}\right) \frac{\partial^{2}}{\partial z_{e} \partial r_{e h}}-\frac{1}{2} \frac{\partial^{2}}{\partial z_{e}^{2}} .
\end{aligned}
$$

The hole kinetic energy operator $T_{h}$ is obtained by interchanging the index $e$ and $h$.

The quantum well potential is defined by

$$
V_{w}=V_{w}^{e}+V_{w}^{h},
$$

where $V_{w}^{e}$ and $V_{w}^{h}$ are the electron and hole confinement potentials. For a SQD embedded in a glass matrix, the hypothesis of infinitely deep electron and hole potentials is perfectly valid, so we can write

$$
V_{w}^{i}=\left\{\begin{array}{ll}
0 ; & \text { if } r_{i} \leq R \\
+\infty ; & \text { if } r_{i}>R
\end{array} i=(e, h) .\right.
$$

We can remark that in the case of a SQD surrounded by another semiconductor the validity of this approximation depends on the conduction and valence band offsets as well as on the radius $R$. We note that many studies have demonstrated that for the intermediate to low quantum confinement $\left(R \geq 1 a^{*}\right)$, the total energy of the complex is not very sensitive to the potential model. ${ }^{11}$

The electric energy operator is given by

$$
W=f\left(z_{e}-z_{h}\right),
$$

where $z_{e}$ and $z_{h}$ are $z$-coordinates of the electron and hole. We have introduced the dimensionless parameter $f=\frac{F}{F_{i}}$ where $F_{i}=\frac{R^{*}}{2 a^{*}} . F_{i}$ is twice the donor ionization field defined by Blossey. ${ }^{28}$

The energy $E_{\left(D^{+}, X\right)}$ and the corresponding envelope wave function are solutions of the Schrödinger equation 


$$
H \Psi\left(r_{e}, r_{h}, r_{e h}, z_{e}, z_{h}\right)=E_{\left(D^{+}, X\right)} \Psi\left(r_{e}, r_{h}, r_{e h}, z_{e}, z_{h}\right) .
$$

This equation cannot be solved analytically, so the ground state solution is determined using a variational method. The ground state energy of the system is obtained by minimizing the expectation value of $H$

$$
E_{\left(D^{+}, X\right)}=\frac{\langle\Psi|H| \Psi\rangle}{\langle\Psi \mid \Psi\rangle}
$$

Our method is based on the development of the wave function and energy in series of powers of the electric field strength $f$. If we restrict our calculations to the second order of the development, the trial wave function is written as follows:

$$
|\Psi\rangle=\left|\Psi_{0}\right\rangle+f\left|\Psi_{1}\right\rangle+f^{2}\left|\Psi_{2}\right\rangle+\cdots .
$$

The ground state wave function of the $\left(D^{+}, X\right)$ complex at zero field $(f=0)$ is taken as

$$
\begin{aligned}
\left|\Psi_{0}\right\rangle= & \sum_{m n p} C_{m n p} j_{0}\left(\frac{\pi r_{e}}{R}\right) j_{0}\left(\frac{\pi r_{h}}{R}\right) r_{e}^{m} r_{h}^{n} r_{e h}^{p} \\
& \times \exp \left(-\eta r_{e}\right) \exp \left(-\beta r_{e h}\right) .
\end{aligned}
$$

In order to account for the electrical distortion of symmetry, we complete $\left|\Psi_{0}\right\rangle$ by

$$
\begin{aligned}
& \left|\Psi_{1}\right\rangle=a\left(z_{e}-z_{h}\right)\left|\Psi_{0}\right\rangle, \\
& \left|\Psi_{2}\right\rangle=b\left(z_{e}-z_{h}\right)^{2}\left|\Psi_{0}\right\rangle,
\end{aligned}
$$

where $a$ and $b$ are variational parameters. $j_{0}\left(q r_{i}\right)=\sin \left(q r_{i}\right) /$ $q r_{i}\left(q=\frac{\pi}{R}\right.$ and $\left.i=e, h\right)$, is the zero-order spherical Bessel function. ${ }^{29}$ The product $r_{e}^{m} r_{h}^{n} r_{e h}^{p}$ takes into account the spatial correlations between the different particles; the integers $m$, $n$, and $p$ are positive or equal to zero. The first exponential factor $e^{-\eta r_{e}}$ describes the Coulomb spatial correlation between the electron and the ionized donor while the second exponential $e^{-\beta r_{e h}}$ describes the Coulomb spatial correlation between the electron and the hole. In our previous works, ${ }^{23}$ we have shown that this kind of correlated trial wave function including the two non-linear coefficients $\eta$ and $\beta$ and developed on a large number of terms allowed to obtain satisfactory results in the determination of fundamental energy of excitonic complexes like charged exciton or ionized donor bound exciton. With the same manner in expression (10), the expansion of the energy in powers of electric field strength can be written as

$$
E=E_{0}+E_{1} f+E_{2} f^{2} .
$$

This expression has the form $E=E_{0}-\mu f-\alpha f^{2}$, where $\mu$ and $\alpha$ are, respectively, the component of the permanent dipole moment and the polarizability in the field direction, thus we have $E_{1}=-\mu$ and $E_{2}=-\alpha$. We draw attention to the fact that in the general case the polarizability is a tensor and is reduced to a scalar in the case of an isotropic system.

Taking into account the previous hypothesis the expression of $\langle\Psi \mid \Psi\rangle$ takes the form

$$
\langle\Psi \mid \Psi\rangle=N_{00}+f N_{11}+f^{2} N_{22}
$$

with

$$
\begin{gathered}
N_{00}=\left\langle\Psi_{0} \mid \Psi_{0}\right\rangle, \\
N_{11}=2\left\langle\Psi_{0} \mid \Psi_{1}\right\rangle, \\
N_{22}=\left\langle\Psi_{1} \mid \Psi_{1}\right\rangle+2\left\langle\Psi_{2} \mid \Psi_{0}\right\rangle,
\end{gathered}
$$

while the expression of $\langle\Psi|H| \Psi\rangle$ becomes

$$
\langle\Psi|H| \Psi\rangle=H_{00}+f H_{11}+f^{2} H_{22},
$$

such as

$$
\begin{gathered}
H_{00}=\left\langle\Psi_{0}\left|H_{0}\right| \Psi_{0}\right\rangle \\
H_{11}=\left\langle\Psi_{1}\left|H_{0}\right| \Psi_{0}\right\rangle+\left\langle\Psi_{0}\left|H_{0}\right| \Psi_{1}\right\rangle+\left\langle\Psi_{0}\left|\left(z_{e}-z_{h}\right)\right| \Psi_{0}\right\rangle \\
H_{22}=\left\langle\Psi_{0}\left|H_{0}\right| \Psi_{2}\right\rangle+\left\langle\Psi_{1}\left|H_{0}\right| \Psi_{1}\right\rangle+\left\langle\Psi_{2}\left|H_{0}\right| \Psi_{0}\right\rangle \\
+\left\langle\Psi_{0}\left|\left(z_{e}-z_{h}\right)\right| \Psi_{1}\right\rangle+\left\langle\Psi_{1}\left|\left(z_{e}-z_{h}\right)\right| \Psi_{0}\right\rangle .
\end{gathered}
$$

For a weak electric field, the Taylor expansion of expression (7) leads to

$$
\begin{aligned}
E_{\left(D^{+}, X\right)}= & \frac{H_{00}+H_{11} f+f^{2} H_{22}}{N_{00}+N_{11} f+f^{2} N_{22}} \\
= & {\left[\frac{H_{00}}{N_{00}}+\left(\frac{H_{11}}{N_{00}}-\frac{H_{00}}{N_{00}^{2}} N_{11}\right) f\right.} \\
& \left.-f^{2}\left(\frac{H_{00}}{N_{00}}\left(\frac{1}{N_{00}} N_{22}-\frac{1}{N_{00}^{2}} N_{11}^{2}\right)-\frac{H_{22}}{N_{00}}+\frac{H_{11}}{N_{00}^{2}} N_{11}\right)\right] .
\end{aligned}
$$

In order to calculate the different integrals, we use the elementary volume in Hylleraas coordinates

$$
d v=\frac{8 \pi r_{e} r_{h} r_{e h} d r_{e} d r_{h} d r_{e h} d z_{e} d z_{h}}{Q}
$$

where the denominator $Q$ is given by

$$
Q=\sqrt{4\left(r_{e}^{2}-z_{e}^{2}\right)\left(r_{h}^{2}-z_{h}^{2}\right)-\left(r_{e}^{2}+r_{h}^{2}-r_{e h}^{2}-2 z_{e} z_{h}\right)^{2}} .
$$

The integrations over $z_{e}$ and $z_{h}$ were performed by generalization of Atanasoff integrals ${ }^{25}$ and done in domains $-r_{e} \leq z_{e} \leq r_{e}$ and $Z_{1} \leq z_{h} \leq Z_{2}$, where $Z_{1}$ and $Z_{2}$ are the roots of $Q$. In these conditions and because of the parity of the wave function on $z_{e}$ and $z_{h}$, we can easily verify that the integrals $\left\langle\Psi_{0} \mid \Psi_{1}\right\rangle,\left\langle\Psi_{1} \mid \Psi_{0}\right\rangle, N_{11}$, and $H_{11}$ vanish, which leads to a zero coefficient of polarization $(\mu=0)$. Thus, the expression of the total energy reduces to

$$
E=\frac{H_{00}+f^{2} H_{22}}{N_{00}+f^{2} N_{22}} .
$$

For a weak electric field, the Taylor expansion of expression (27) leads to

$$
E=E_{0}+f^{2} E_{2}=E_{0}-\alpha f^{2}=\frac{H_{00}}{N_{00}}+f^{2}\left(\frac{H_{22}}{N_{00}}-\frac{H_{00}}{N_{00}^{2}} N_{22}\right) .
$$


We recall that the conditions $\frac{\partial E}{\partial C_{\text {mup }}}=0$ for all possible values of $m, n, p$, and the minimization with respect to $\eta, \beta, a$, and $b$ lead to determine the energy without electric field $E_{0}$ and the polarizability $\alpha$ deduced from relation (28)

$$
E_{0}=\frac{H_{00}}{N_{00}} \quad \text { and } \quad \alpha=-E_{2}=-\left(\frac{H_{22}}{N_{00}}-\frac{H_{00}}{N_{00}^{2}} N_{22}\right) .
$$

In order to test our approach, we apply the same formalism to determine the exciton energy without electric field. In this case, the Coulomb potential is reduced to $V_{c}=-1 / r_{e h}$ and we choose an excitonic wave function for the unperturbed case

$$
\left|\Psi_{X}\right\rangle=\sum_{m n p} C_{m n p} j_{0}\left(\frac{\pi r_{e}}{R}\right) j_{0}\left(\frac{\pi r_{h}}{R}\right) r_{e}^{m} r_{h}^{n} r_{e h}^{p} \exp \left(-\beta r_{e h}\right)
$$

\section{RESULTS AND DISCUSSIONS}

In order to validate our calculations, we apply this approach to the case of single exciton (without ionized donor) and compare our results at $F=0$ with some existing data. We start our analysis with some results for $\mathrm{ZnO}$. In Fig. 1, the solid line presents the variations of our calculated band gap energy defined as $E_{g}^{*}=E_{g}^{b u l k}+E_{X}$ as a function of the dot radius, where $E_{g}^{b u l k}$ is the band gap energy of the bulk material and $E_{X}$ is the ground energy of the exciton obtained by minimizing the expression (9), taking into account the excitonic wave function given by the expression (30). We note that our theoretical results are obtained for heavy hole mass $m_{h h}^{*}=0.79 m_{0}$, electron mass $m_{e}^{*}=0.24 m_{0}$, and dielectric constant $\varepsilon=6.4$. This choice of physical

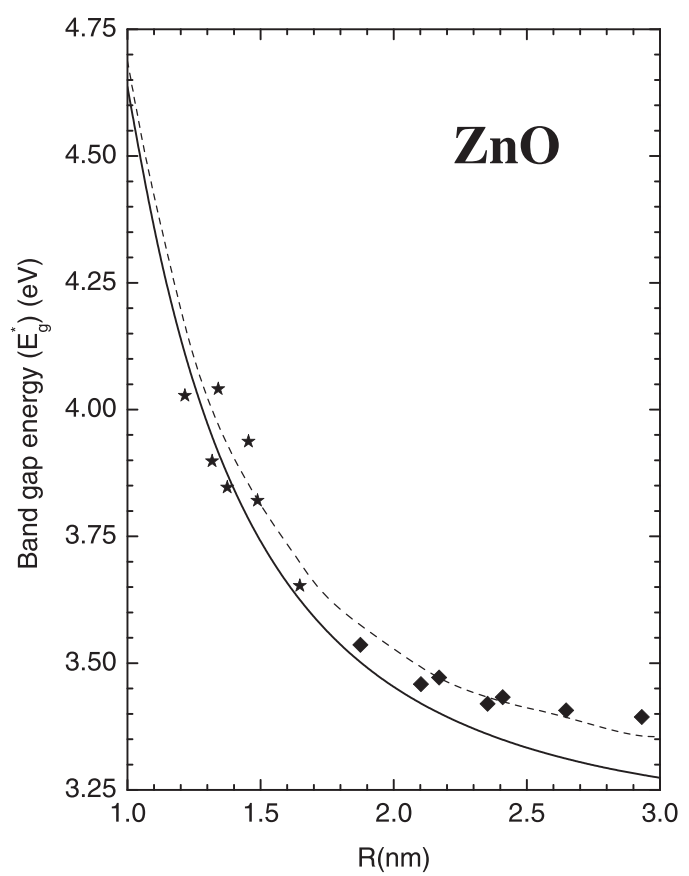

FIG. 1. Variation of band gap energy $E_{g}^{*}=E_{g}^{b u l k}+E_{X}$ as function of the dot radius, for $\mathrm{ZnO}$. The solid line indicates the results of the present model with $m_{h h}=0.79 m_{0}$; the dashed line represents the results obtained from $k p$ method; ${ }^{6}$ the stars and squares are the experimental results. ${ }^{31,32}$ parameters is used here in order to respect the well known bulk $\mathrm{ZnO}$ binding energy $(\sim 60 \mathrm{meV}) .{ }^{30}$ Our theoretical predictions of the band gap are in good agreement with existing experimental data (stars and squares) given in references. ${ }^{31,32}$ The dependence of the optical gap for this set of radii can be fitted by $E_{g}^{*}=3.44+1.3 R^{-2.5}$. The dashed lines refer to Fonoberov and Balandin ${ }^{6}$ calculations based on the $k p$ method using an heavy hole $m_{h h}^{*}=2.31 m_{0}$. A simple reading of all of these results shows that the calculated photo-luminescence transition depends strongly on the choice of the value of the heavy hole mass in $\mathrm{ZnO}$ and the dielectric constant. Unfortunately, the $\mathrm{ZnO}$ heavy hole mass value is actually subject to some controversies due to the different measurement techniques employed. This value ranges between $0.59 m_{0}$ and $2.31 m_{0}$. For more details, see Refs. 12, 23, and 33. However, we point out that the dependence of the dielectric constant on the dot size considerably affects the photo-luminescence transition. More recently, the experimental study initiated by Yang et al. ${ }^{34}$ shows that in $\mathrm{ZnO}$, the dielectric constant ranges from 2.7 to 6.4 and varies according to a law defining its behavior as function of the dots sizes. A very interesting result to mention is that announced by S. Baskoutas and G. Bester: ${ }^{35}$ within atomistic empirical pseudopotential, they calculated the electronic and the optical properties of Wurtzite $\mathrm{ZnO}$ QDs larger than $2.6 \mathrm{~nm}$ with several thousand atoms and including all relevant effects such as multi-band coupling and spin orbit interactions. They showed that the dependence of the optical gap can be fitted by $E_{g}^{*}=3.44+1.3 D^{-1.17}$ for diameter values between $1.6 \mathrm{~nm}$ and $3.6 \mathrm{~nm}$. In the same context, we report in Fig. 2, our calculation of the band gap transition of exciton in a $\mathrm{PbSe}$ SQD. In the same figure, we remind some

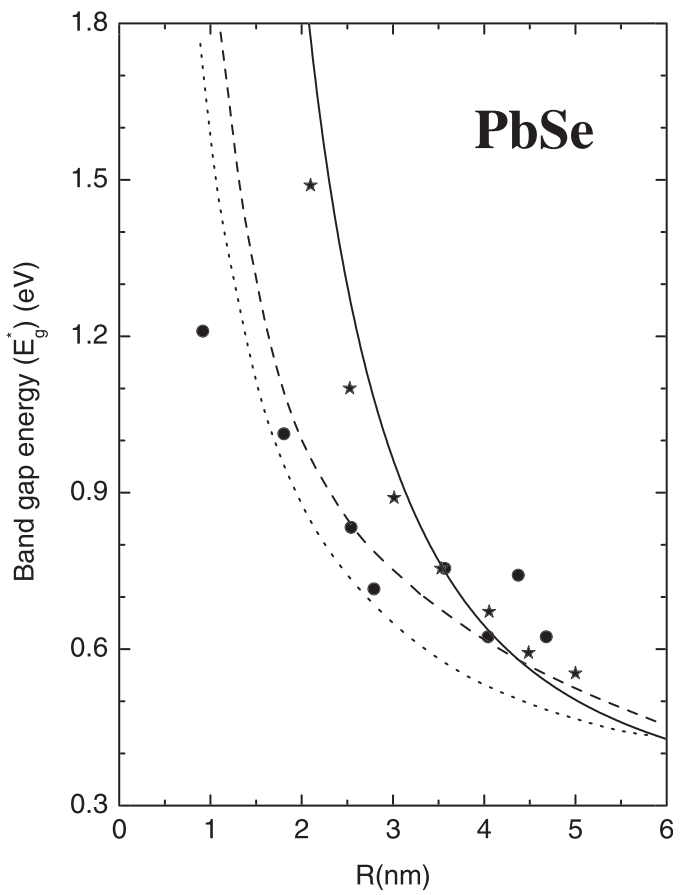

FIG. 2. Comparison of the variations of band gap energy $E_{g}^{*}=E_{g}^{b u l k}+E_{X}$ versus the dot radius, for $\mathrm{PbSe}$ obtained by different method: PMM (dashed line), ${ }^{36}$ first order perturbation (doted line), ${ }^{37} \mathrm{kp}$-method (stars), ${ }^{38}$ experimental data (circles). ${ }^{39}$ The solid line indicates the results of the present formalism. 
TABLE I. Electron mass, hole mass, $\sigma=m_{e}^{*} / m_{h}^{*}$, dielectric constant, $a_{D}$ and $R^{*}=2 R_{D}$, and field ionization for three materials: InAs, ZnO, and $P b S e$.

\begin{tabular}{lcccccccc}
\hline \hline & $m_{e}^{*} / m_{0}$ & $m_{h}^{*} / m_{0}$ & $\sigma$ & $\varepsilon_{\infty}$ & $E_{g}(\mathrm{eV})$ & $a_{D}(\mathrm{~nm})$ & $R^{*}(\mathrm{meV})$ & $F_{i}\left(\mathrm{kV} \cdot \mathrm{cm}^{-1}\right)$ \\
\hline $\mathrm{InAs}^{40}$ & 0.023 & 0.57 & 0.04 & 1.85 & 0.36 & 28.46 & 4.02 & 1.51 \\
$\mathrm{ZnO}^{30}$ & 0.24 & 0.79 & 0.30 & 6.4 & 3.44 & 1.41 & 159.44 & 1129.88 \\
$\mathrm{PbSe}^{41}$ & 0.24 & 0.14 & 1.85 & 23 & 0.28 & 4.68 & 13.37 & 28.57 \\
\hline \hline
\end{tabular}

interesting theoretical results: Potential Morphing Method (dashed line), ${ }^{36}$ first order perturbation (doted line), ${ }^{37} \boldsymbol{k} \boldsymbol{p}$ method (stars) ${ }^{38}$ and experimental data (circles) obtained by Lipovskii et al. ${ }^{39}$ We remark that the results of the present study are close to the $\boldsymbol{k} \boldsymbol{p}$ method and approximately fit the experimental data mainly for $R>2 \mathrm{~nm}$. According to the previous comparisons deduced from Figures 1 and 2, we can assert that our variational-perturbative method using a correlated wave function containing the non linear coefficients is more appropriate for quantitative description of the fundamental state of the excitons and constitute a good mean for the determination of the $\left(D^{+}, X\right)$ energy.

In what follows, we generalize our method for the $\left(D^{+}, X\right)$ complex by analyzing the effect of the localized ionized donor on the confined Stark shift of excitons for three semiconductors materials with three significant masses ratios: InAs $(\sigma=0.04), \mathrm{ZnO}(\sigma=0.30)$, and $\mathrm{PbSe}(\sigma=1.27)$. The full used physical parameters data of these materials are listed in Table I.

In Fig. 3, we plot the ground state energy at zero electric field for SQDs of InAs, ZnO, and PbSe. As we can remark, we retrieve the well known behavior of $\left(D^{+}, X\right){ }^{5,23}$ The energy decreases as the radius increases and reaches the limit $0.5 R^{*}$ for large dot sizes. For a given radius, the energy

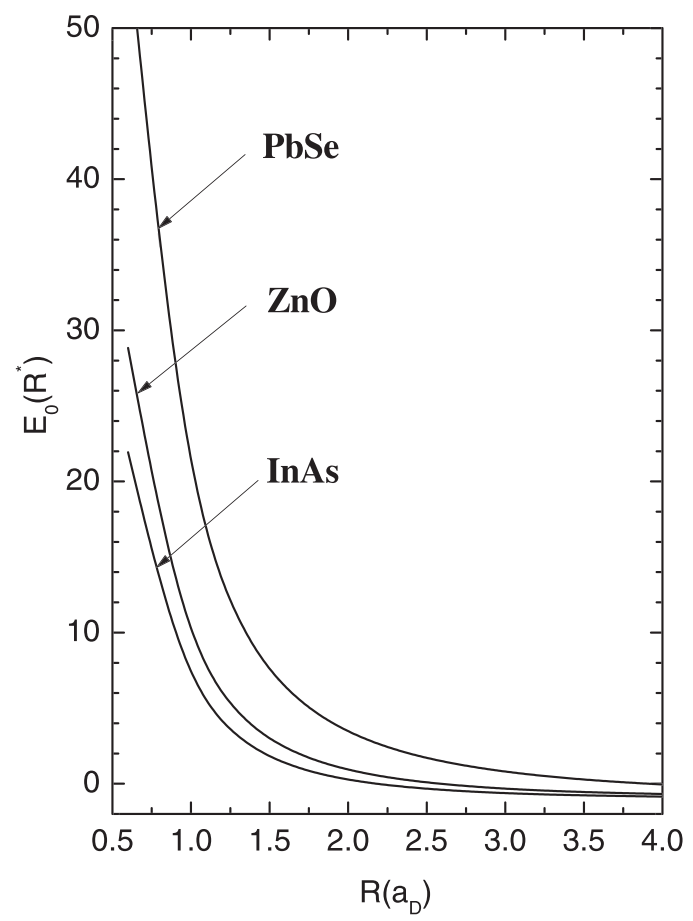

FIG. 3. Variation of the ground state energy of exciton bound to an ionized donor $\left(D^{+}, X\right)$ versus the SQD radius at zero electric field for InAs $(\sigma=0.04), \mathrm{ZnO}(\sigma=0.30)$, and $\mathrm{PbSe}(\sigma=1.27)$. increases with increasing effective mass ratio. In Fig. 4, we plot the polarizability of $\left(D^{+}, X\right)$ given by the relation (22) versus the dot radius. The Stark shift is related to the polarizability by the relation $\Delta E=E(0)-E(F)=\alpha f^{2}$. We remark that the polarizability is more pronounced for the materials with low mass ratio and increases with increasing size dots. The monotonic and positive curvature behavior of the polarizability has allowed us to establish a law of variation of this behavior as function of the dots sizes. We have fitted our results using the relationship $g R^{\gamma}$, and we determined the values of the coefficients $g$ and $\gamma$ for the three considered materials. Our calculations reveal an important conclusion: the polarizability $\alpha$ scales proportionally to $R^{3.5}\left(\alpha \sim R^{3.5}\right)$. A similar behaviour was observed by Wang et al. ${ }^{42}$ in measurements of the exciton polarizability in $C d S e$ nanocrystals. The full results of the fit for the three materials are regrouped in Table II.

Thus, the Stark shift can be estimated by the product of $R^{3.5}$ and $f^{2}$ with a factor $k$ which depends on the materials nature $\left(\Delta E=\alpha f^{2}=k R^{3.5} f^{2}\right)$. We note that this method remains valid only in the weak electric fields cases $(f \ll 1)$. We underline here that our simple approach allows to calculate

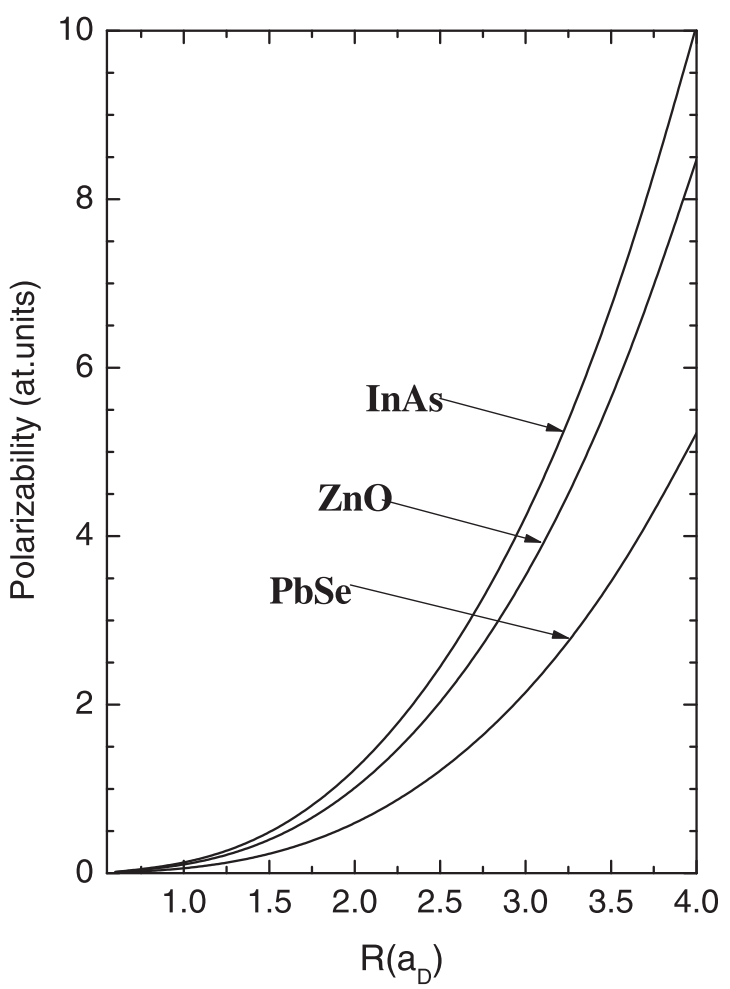

FIG. 4. Variation of the polarizability $\alpha\left(\alpha=-E_{2}\right)$ of an exciton bound to an ionized donor $\left(D^{+}, X\right)$ versus the SQD radius for InAs $(\sigma=0.04), \mathrm{ZnO}$ $(\sigma=0.30)$, and $\mathrm{PbSe}(\sigma=1.27)$. 
TABLE II. Theoretical prediction of the behavior of the polarizabilities of exciton bound to an ionized donor $\left(D^{+}, X\right)$ as function of the SQD radius for InAs $(\sigma=0.04), \mathrm{ZnO}(\sigma=0.30)$, and $\mathrm{PbSe}(\sigma=1.27)$.

\begin{tabular}{cccc}
\hline \hline & InAs & ZnO & PbSe \\
\hline$\alpha$ & $0.032 R^{3.47}$ & $0.03 R^{3.52}$ & $0.027 R^{3.46}$ \\
\hline \hline
\end{tabular}

easily the energy of $\left(D^{+}, X\right)$ submitted to an electric field. Indeed, from Fig. 3 and for a given radius, we can extract the energy at zero electric field, and from Fig. 4, we extract the polarizability for the same radius and we use the relation (21) to determine the energy - whatever the electric field strength. In the aim to facilitate the comprehension of optical response in electroluminescence experiences of $\left(D^{+}, X\right)$, we complete our discussion by comparing the polarizabilities of neutral donor $\left(D^{0}\right)$, exciton $(X)$, and exciton bound to an ionized donor $\left(D^{+}, X\right)$. Theoretical predictions of polarizabilities for the three semiconductors material $\operatorname{InAs}, \mathrm{ZnO}$, and $\mathrm{PbSe}$ are given in Table III for the three regimes of confinement. As we can see, for all types of confinement, the polarizability of exciton is situated between that of the impurity and that of the $\left(D^{+}, X\right), \alpha_{D^{0}}<\alpha_{X}<\alpha_{\left(D^{+}, X\right)}$. All these results clearly show that the donor center affects the binding energy of exciton and facilitates the dissociation process in electric field.

Let us turn to another interesting point which is the relation between the energy of $\left(D^{+}, X\right)$ and $D^{0}$. Indeed, in a great variety of semiconductors, the binding energy of excitons bound to ionized donor shows a linear dependence on neutral impurities. This variation is described in the form of a generalized Haynes' rule $E_{\left(D^{+}, X\right)}=A E_{D^{0}}+B(\mathrm{meV})$; the constants $A$ and $B$ are to be determined by fitting the curve $E_{\left(D^{+} X\right)}$ as function as $E_{D^{0}}$ for different radii. Our theoretical results show that in the limit of weak electric field, the energy of the $\left(D^{+}, X\right)$ fits linearly as function of the donor energy accordingly the Haynes' rule. Table IV gives the Haynes coefficients $A$ and $B$ for different electric strength.

\section{CONCLUSION}

We have studied the effect of an external electric field on an exciton bound to an ionized donor confined in a spherical quantum dot embedded in a glass matrix and described by an hard well. By using a variational-perturbative method

TABLE III. Comparison of the polarizabilities of an exciton bound to an ionized donor $\left(D^{+}, X\right)$, exciton $X$, and donor $D^{0}$ for $I n A s, Z n O$, and $P b S e$.

\begin{tabular}{ccccc}
\hline \hline$R\left(a_{D}\right)$ & $\alpha\left(\mathrm{eV} \cdot \mathrm{cm}^{2} \cdot \mathrm{kV}^{-2}\right)$ & InAs & ZnO & PbSe \\
\hline$R=1$ & $\alpha_{D^{0}}$ & $0.3797 \times 10^{-4}$ & $1.8823 \times 10^{-6}$ & $9.3124 \times 10^{-6}$ \\
& $\alpha_{X}$ & $1.1449 \times 10^{-4}$ & $5.2620 \times 10^{-6}$ & $16.573 \times 10^{-6}$ \\
& $\alpha_{\left(D^{+}, X\right)}$ & $2.8308 \times 10^{-4}$ & $11.347 \times 10^{-6}$ & $34.069 \times 10^{-6}$ \\
$R=2$ & $\alpha_{D^{0}}$ & $4.4549 \times 10^{-4}$ & $2.2086 \times 10^{-5}$ & $10.926 \times 10^{-5}$ \\
& $\alpha_{X}$ & $9.9992 \times 10^{-4}$ & $5.3897 \times 10^{-5}$ & $21.404 \times 10^{-5}$ \\
& $\alpha_{\left(D^{+}, X\right)}$ & $29.714 \times 10^{-4}$ & $13.063 \times 10^{-5}$ & $42.422 \times 10^{-5}$ \\
$R=4$ & $\alpha_{D^{0}}$ & $2.8029 \times 10^{-3}$ & $1.3896 \times 10^{-4}$ & $6.8746 \times 10^{-4}$ \\
& $\alpha_{X}$ & $3.6185 \times 10^{-3}$ & $2.5688 \times 10^{-4}$ & $20.109 \times 10^{-4}$ \\
& $\alpha_{\left(D^{+}, X\right)}$ & $21.366 \times 10^{-3}$ & $11.812 \times 10^{-4}$ & $43.514 \times 10^{-4}$ \\
\hline \hline
\end{tabular}

TABLE IV. Haynes rule $E_{\left(D^{+} X\right)}=A E_{D^{0}}+B$ (meV) for three values of electric field strength and for $\operatorname{InAs}, \mathrm{ZnO}$, and $\mathrm{PbSe}$.

\begin{tabular}{cccc}
\hline \hline & InAs & ZnO & PbSe \\
\hline$F=0$ & $A=1.0718$ & $A=0.5477$ & $A=2.31967$ \\
& $B=0.2059$ & $B=1.3634$ & $B=1.656$ \\
$F=0.5$ & $A=1.0832$ & $A=1,3764$ & $A=2.327$ \\
& $B=-0.888$ & $B=-0.6997$ & $B=-0.6865$ \\
$F=1$ & $A=1.1217$ & $A=1.4196$ & $A=2.351$ \\
& $B=-4.1999$ & $B=-4.4744$ & $B=-2.2379$ \\
\hline \hline
\end{tabular}

and developments of the wave function and the energy in series of powers of electric field, we have first applied this approach to an exciton in order to retrieve and compare with some existing results and we have calculated the energy of $\left(D^{+}, X\right)$ in the presence of the electric field for several materials $\mathrm{ZnO}, \mathrm{PbSe}$, and InAs, which possess different electron to hole masses ratios. The simplicity of the present approach allows the calculation of energy in the presence of an electric field by extracting the two quantities: the energy without electric field $E_{0}$ and the second term of the development $E_{2}$. Our theoretical prediction shows that the polarizability of $\left(D^{+}, X\right)$ varies proportionally to $R^{3.5}$ and follows an ordering $\alpha_{D^{0}}<\alpha_{X}<\alpha_{\left(D^{+} X\right)}$. The last point to highlight is that the Haynes rule remains valid even in the presence of a weak electric field. We hope that the simplicity of this contribution opens the way to investigate these excitonic complexes because the great role of these systems in the photonic emissions intervening in quantum bit devices.

\section{ACKNOWLEDGMENTS}

This work was supported by the Research Project "PHC MAGHREB, MAG/NRG/14, Campus France, N³2595SL".

${ }^{1}$ M. A. Lampert, Phys. Rev. Lett. 1, 450 (1958).

${ }^{2}$ T. Skettrup, M. Suffczynski, and W. Gorzkowski, Phys. Rev. B 4, 512 (1971).

${ }^{3}$ L. Stauffer and B. Stébé, Phys. Rev. B 39, 5345 (1989).

${ }^{4}$ B. Stébé and L. Stauffer, Superlatt. Microstruct. 5, 451 (1989).

${ }^{5}$ B. Stébé, E. Assaid, F. Dujardin, and S. Le Goff, Phys. Rev. B 54, 17785 (1996).

${ }^{6}$ V. A. Fonoberov and A. A. Balandin, Appl. Phys. Lett. 85, 5971 (2004).

${ }^{7}$ V. A. Fonoberov, E. P. Pokatilov, V. M. Fomin, and J. T. Devreese, Phys. Rev. Lett. 92, 127402 (2004).

${ }^{8}$ V. A. Fonoberov, E. P. Pokatilov, and A. A. Balandin, Phys. Rev. B 66, 085310 (2002).

${ }^{9}$ S. Baskoutas, W. Schommers, A. F. Terzis, V. Kapaklis, M. Rieth, and C. Politis, Phys. Lett. A 308, 219 (2003).

${ }^{10}$ W. Xie and J. Gu, Physica B 337, 58 (2003).

${ }^{11}$ A. A. Avetisyan, A. P. Djotyan, and E. M. Kazaryan, Phys. Status Solidi C 0, 734 (2003)

${ }^{12}$ B. K. Meyer, J. Sann, S. Lautenschläger, M. R. Wagner, and A. Hoffmann, Phys. Rev. B 76, 184120 (2007).

${ }^{13}$ D. A. B. Miller, D. S. Chemla, T. C. Damen, A. C. Gossard, W. Wiegmann, T. H. Wood, and C. A. Burrus, Phys. Rev. Lett. 53, 2173 (1984).

${ }^{14}$ G. W. Wen, J. Y. Lin, H. X. Jiang, and Z. Chen, Phys. Rev. B 52, 5913 (1995).

${ }^{15}$ S. Nomura and T. Kobayashi, Solid State Commun. 74, 1153 (1990).

${ }^{16}$ S. A. Empedocles and M. G. Bawendi, Science 278, 2114 (1997).

${ }^{17}$ E. Feddi, E. Assaid, F. Dujardin, B. Stébé, and J. Diouri, Phys. Scr. 62, 88 (2000).

${ }^{18}$ E. Feddi, M. El Haouari, E. Assaid, B. Stébé, J. El Khamkhami, and F. Dujardin, Phys. Status Solidi B 240, 106 (2003). 
${ }^{19}$ F. Dujardin, A. Oukerroum, E. Feddi, J. Bosch Bailach, J. Mart ínezPastor, and M. Zazi, J. Appl. Phys. 111, 034317 (2012).

${ }^{20}$ F. Dujardin, E. Feddi, A. Oukerroum, J. Bosch Bailach, J. Mart ínezPastor, and E. Assaid, J. Appl. Phys. 113, 064314 (2013).

${ }^{21}$ A. Oukerroum, E. Feddi, J. Bosch Bailach, J. Martínez-Pastor, F. Dujardin, and E. Assaid, J. Phys.: Condens. Matter 22, 375301 (2010).

${ }^{22}$ E. Niculescu, L. Burileanu, and I. Sandulescu, J. Optoelectron. Adv. Mater. 7, 2775 (2005).

${ }^{23}$ F. Dujardin, E. Feddi, E. Assaid, and A. Oukerroum, Eur. Phys. J. B 74, 507 (2010).

${ }^{24}$ L. Shi and Z. Yan, J. Appl. Phys. 114, 194301 (2013).

${ }^{25}$ J. V. Atanasoff, Phys. Rev. B 36, 1232 (1930).

${ }^{26}$ M. Katih, J. Diouri, and E. Feddi, Phys. Status Solidi B 175, 349 (1993).

${ }^{27}$ E. A. Hylleraas, Z. Phys. 48, 469 (1928).

${ }^{28}$ D. F. Blossey, Phys. Rev. B 2, 3976 (1970).

${ }^{29}$ Handbook of Mathematical Functions, edited by M. Abramowitz and I. A. Stegun (Dover, New York, 1972).

${ }^{30}$ C. Morhain, T. Bretagnon, P. Lefebvre, X. Tang, P. Valvin, T. Guillet, B. Gil, T. Taliercio, M. Teisseire-Doninelli, B. Vinter, and C. Deparis, Phys. Rev. B 72, 241305(R) (2005).

${ }^{31}$ E. A. Meulenkamp, J. Phys. Chem. B 102, 5566 (1998).
${ }^{32}$ A. Wood, M. Giersig, M. Hilgendorff, A. Vilas-Campos, L. M. LizMarzán, and P. Mulvaney, Aus. J. Chem. 56, 1051 (2003).

${ }^{33}$ W. R. L. Lambrecht, A. V. Rodina, S. Limpijumnong, B. Segall, and B. K. Meyer, Phys. Rev. B 65, 075207 (2002).

${ }^{34}$ Y. Yang, W. Guo, X. Wang, Z. Wang, J. Qi, and Y. Zhang, Nano Lett. 12, 1919 (2012).

${ }^{35}$ S. Baskoutas and G. Bester, J. Phys. Chem. C 114, 9301 (2010).

${ }^{36}$ S. Baskoutas, A. F. Terzis, and W. Schommers, J. Comput. Theor. Nanosci. 3, 269 (2006).

${ }^{37}$ G. Pellegreni, G. Mattei, and P. Mazzoldi, J. Appl. Phys. 97, 073706 (2005).

${ }^{38}$ G. E. Tudury, M. V. Marquezini, L. G. Ferreira, L. C. Barbosa, and C. L. Cesar, Phys. Rev. B 62, 7357 (2000).

${ }^{39}$ A. Lipovskii, E. Kolobkova, V. Petrikov, I. Kang, A. Olkhovets, T. Krauss, M. Thomas, J. Silcox, F. Wise, and Q. Shen, Appl. Phys. Lett. 71, 3406 (1997).

${ }^{40}$ Semiconductor Data Handbook, edited by O. Madelung, 3rd ed. (SpringerVerlag, Berlin, 2004).

${ }^{41}$ G. Zohar, R. Baer, and E. Rabani, J. Phys. Chem. Lett. 4, 317 (2013).

${ }^{42}$ F. Wang, J. Shan, M. A. Islam, I. P. Herman, M. Bonn, and T. F. Heinz, Nat. Mater. 5, 861 (2006). 